Full paper

\title{
Two-dimensional mesoporous vanadium phosphate nanosheets through liquid crystal templating method toward supercapacitor application
}

\author{
Peng Mei ${ }^{\mathrm{a}}$, Yusuf Valentino Kaneti ${ }^{\mathrm{a}, \mathrm{b}}$, Malay Pramanik ${ }^{\mathrm{a}}$, Toshiaki Takei ${ }^{\mathrm{a}}$, Ömer Dag ${ }^{\mathrm{c}}$, \\ Yoshiyuki Sugahara ${ }^{\mathrm{d}}$, Yusuke Yamauchi ${ }^{\mathrm{b}, \mathrm{ef}, \mathrm{f},}$ \\ a International Center for Materials Nanoarchitectonics (WPI-MANA), National Institute for Materials Science (NIMS), 1-1 Namiki, Tsukuba, Ibaraki 305-0044, Japan \\ ${ }^{\mathrm{b}}$ College of Chemistry and Molecular Engineering, Qingdao University of Science and Technology, Qingdao 266042, China \\ ${ }^{\mathrm{c}}$ Department of Chemistry, UNAM-National Nanotechnology Research Center, and Institute of Material Science and Nanotechnology, Bilkent University, 06800 Ankara, \\ Turkey \\ d Department of Nanoscience and Nanoengineering, Faculty of Science and Engineering, Waseda University, 3-4-1 Okubo, Shinjuku, Tokyo 169-8555, Japan \\ e School of Chemical Engineering and Australian Institute for Bioengineering and Nanotechnology (AIBN), The University of Queensland, Brisbane, QLD 4072, Australia \\ ${ }^{\mathrm{f}}$ Department of Plant \& Environmental New Resources, Kyung Hee University, 1732 Deogyeong-daero, Giheung-gu, Yongin-si, Gyeonggi-do 446-701, South Korea
}

\section{A R T I C L E I N F O}

\section{Keywords:}

Metal phosphates

Mesoporous materials

Nanosheets

Supercapacitors

Lyotropic liquid crystals

Templated synthesis

\begin{abstract}
A B S T R A C T
Mesoporous vanadium phosphate $\left(\mathrm{VOPO}_{4}\right)$ nanosheets have been successfully synthesized through an easy and reproducible lyotropic liquid crystals (LLC) templating approach for the first time. Using the triblock copolymer (P123) as a surfactant, $\mathrm{VOPO}_{4}$ precursor with a well-developed 2D hexagonal mesostructure can be obtained. Following complete removal of the template by calcination, crystallized $\mathrm{VOPO}_{4}$ frameworks with less-ordered mesostructure are achieved. The as-prepared mesoporous $\mathrm{VOPO}_{4}$ nanosheets exhibit superior pseudocapacitive performance $\left(767 \mathrm{~F} \mathrm{~g}^{-1}\right.$ at $0.5 \mathrm{~A} \mathrm{~g}^{-1}$ ) by virtue of the favorable mesostructure that gives rise to abundant easily accessible redox active sites as well as reinforced charge transfer and ion diffusion properties. The charge storage mechanism of the mesoporous $\mathrm{VOPO}_{4}$ nanosheets has been experimentally demonstrated to be based on the reversible two-step redox reactions between $\mathrm{V}(\mathrm{V})$ and V(III) in acidic medium. This advantageous LLC templating strategy is expected to open up a new route for designing various mesoporous metal phosphates with superior electrochemical performance for utilization in energy storage devices.
\end{abstract}

\section{Introduction}

Mesoporous architectures have attracted significant interests in material science research due to their enticing abilities to accommodate and interact with nanoscale guest species (e.g., ions, molecules), which endue them with diverse utilities in a vast number of applications ranging from sensors, adsorption/separation, catalysis, biotechnology to energy storage and conversion [1-9]. The pioneering discovery of mesoporous silica in the 1990s has triggered an upsurge of research in the synthesis, characterization and application of mesoporous materials $[10,11]$. Strikingly, the lyotropic liquid crystals (LLC) formed by surfactants at high concentrations, have been proposed as a soft template and provided new ways to enable directed synthesis of new silica- and non-silica-based materials with desirable mesoporous architectures and controllable porosity, which outperform the complicated, costly, and industrially unfeasible hard-template method [12-18]. Despite the enormous advancements made by the LLC method, this method was primarily used to prepare mesoporous silicas, metals, alloys, and few selected metal oxides and sulfides. To the best of our knowledge, the extension of the LLC-templating strategy to the synthesis of mesoporous metal phosphates has not yet been demonstrated and such method may provide great scientific and applicative value.

Vanadium phosphates, a fascinating family of compounds with fertile structural chemistry, have attracted tremendous attention owing to their excellent catalytic activities towards selective oxidation of hydrocarbons and other reactions $[19,20]$. The typical and simplest vanadium phosphate, $\mathrm{VOPO}_{4}$, has recently been discovered to exhibit exciting potential for energy storage devices. Layered $\mathrm{VOPO}_{4}$ compound is potentially attractive for cation (e.g., lithium or sodium) intercalation [21]. With the introduction of more electronegative $\left(\mathrm{PO}_{4}\right)^{3-}$ anion, the vanadium redox couples $\mathrm{V}^{4+} / \mathrm{V}^{5+}$ and/or $\mathrm{V}^{3+} / \mathrm{V}^{4+}$ could provide a higher potential than simple oxides and thereby, enhance the

\footnotetext{
* Corresponding author: School of Chemical Engineering and Australian Institute for Bioengineering and Nanotechnology (AIBN), The University of Queensland, Brisbane, QLD 4072, Australia

E-mail address: y.yamauchi@uq.edu.au (Y. Yamauchi).
} 
energy density of the devices [22]. Therefore, as a prospective electrode material, $\mathrm{VOPO}_{4}$ has attracted extensive research interests for lithium/ sodium-ion batteries (LIBs/SIBs) [23-29]. Nevertheless, the utilization of $\mathrm{VOPO}_{4}$ in other energy storage devices, such as supercapacitors, is rarely explored and the current knowledge in terms of supercapacitive performance and charge storage mechanism of $\mathrm{VOPO}_{4}$ is rather limited [30-33]. While several exquisite two-dimensional (2D) $\mathrm{VOPO}_{4}$ nanosheets have been synthesized [31,33], the absence of pores has restricted them to reach their full potential as electrode materials. It has been well documented that mesoporous architectures can provide some unique advantages for supercapacitor applications, including rapid and easy access to abundant redox active sites arising from the interconnected mesopores [34]. Although there have been several reports on the synthesis of mesostructured vanadium phosphorus oxides [35-37], these compounds generally contain large amount of insulating surfactant residues and non-stoichiometric compositions that render them virtually unattractive for energy storage devices. Therefore, the synthesis of organic-free mesoporous $\mathrm{VOPO}_{4}$ nanosheets as high-performance electrode for supercapacitors, as well as their reaction mechanism study, could be very advantageous for increasing the diversity and power deliverability of current energy storage systems.

Herein, we report the first case of mesoporous $\mathrm{VOPO}_{4}$ nanosheets (with no organic residues) synthesized via a facile and reproducible LLC-templating method. In our proposed method, triblock copolymer (P123) is adopted as a surfactant and a precursor material with welldeveloped 2D hexagonal mesostructure is attained. Upon the complete removal of the template by calcination, $\delta$ - $\mathrm{VOPO}_{4}$ nanosheets with lessordered mesoporous structure and crystallized frameworks are obtained. The as-prepared mesoporous $\mathrm{VOPO}_{4}$ nanosheets exhibit both superior pseudocapacitance and rate capability compared to the bulk counterpart, with comparable performance to some reported vanadium phosphate/graphene composite and metal phosphates. Furthermore, we experimentally demonstrate that the charge storage mechanism of the mesoporous $\mathrm{VOPO}_{4}$ nanosheets is based on the reversible two-step redox reactions between $\mathrm{V}(\mathrm{V})$ and $\mathrm{V}(\mathrm{III})$ in acidic medium.

\section{Experimental section}

\subsection{Materials}

Vanadyl acetylacetonate $\left(\mathrm{VO}\left(\mathrm{C}_{5} \mathrm{H}_{7} \mathrm{O}_{2}\right)_{2}, 98 \mathrm{wt} \%\right)$ and triblock copolymer, poly(ethylene glycol)-block-poly(propylene glycol)-block-poly (ethylene glycol) (Pluronic P123, $M_{\mathrm{n}}=5800$ ) were provided by Sigma Aldrich. Phosphoric acid $\left(\mathrm{H}_{3} \mathrm{PO}_{4}, 85 \mathrm{wt} \%\right)$, hydrochloric acid $(\mathrm{HCl}$, $36 \mathrm{wt} \%)$, sulfuric acid $\left(\mathrm{H}_{2} \mathrm{SO}_{4}, 1 \mathrm{~mol} / \mathrm{L}\right)$ and ethanol were purchased from Nacalai Tesque. All chemical reagents were used as received without further purification.

\subsection{Synthesis}

In a typical synthetic process of mesoporous vanadium phosphate $\left(\mathrm{VOPO}_{4}\right.$, abbreviated as VPO) nanosheets, $0.5 \mathrm{~g}$ of Pluronic P123 was firstly dissolved in $10 \mathrm{~mL}$ of ethanol acidified with $0.66 \mathrm{~g}$ of $\mathrm{HCl}$ solution at room temperature, followed by $2 \mathrm{~h}$ of mild magnetic stirring. Next, $1 \mathrm{mmol}$ of $\mathrm{H}_{3} \mathrm{PO}_{4}$ solution was added into the mixture solution under stirring. In a separate bottle, $1 \mathrm{mmol}$ of $\mathrm{VO}\left(\mathrm{C}_{5} \mathrm{H}_{7} \mathrm{O}_{2}\right)_{2}$ was dissolved in $20 \mathrm{~mL}$ of ethanol under sonication, and was then added dropwise into the above mixture solution. The stirring was continued for another $6 \mathrm{~h}$, and then, the mixture was kept statically in an oven at $60{ }^{\circ} \mathrm{C}$ until blackish green gel-like precursor was formed. Finally, the asobtained gel was calcined in air at $450^{\circ} \mathrm{C}$ for $4 \mathrm{~h}$ to obtain the mesoporous VPO nanosheets. For comparison, bulk VPO material was also prepared in the absence of P123 using similar procedures.

\subsection{Characterizations}

Wide-angle powder X-ray diffraction (XRD) patterns were recorded with a RIGAKU Rint 2000 diffractometer, using monochromatic $\mathrm{Cu}-\mathrm{K} \alpha$ radiation $(40 \mathrm{kV}, 40 \mathrm{~mA})$. Low-angle $\mathrm{XRD}$ patterns were acquired from a RIGAKU SmartLab. Scanning electron microscope (SEM) images were acquired from a Hitachi SU-8000 field emission SEM operated at $5 \mathrm{kV}$. Transmission electron microscope (TEM) observations were conducted on a JEOL JEM-2100F TEM system operated at an accelerating voltage of $200 \mathrm{kV}$. Nitrogen adsorption-desorption analysis was performed using a Belsorp-mini II Sorption System. X-ray photoelectron spectroscopy (XPS) was implemented on a PHI Quantera SXM apparatus. All binding energies were calibrated by referencing to the $\mathrm{C} 1 \mathrm{~s}$ line $(285 \mathrm{eV})$. Fourier transform infrared (FTIR) spectra were collected on a Thermo scientific Nicolet 4700 spectrometer. Thermogravimetric analyses (TGA) were carried out on a Hitachi HT-Seiko Instrument with a heating rate of $3^{\circ} \mathrm{C} \mathrm{min}^{-1}$ in air.

\subsection{Electrochemical measurements}

All the electrochemical measurements in this work were performed using an electrochemical workstation (CHI 660E, CH Instruments, USA) with a standard three-electrode system with platinum electrode as the counter electrode and saturated calomel electrode as the reference electrode. The working electrode was fabricated by casting the slurry composed of $80 \mathrm{wt} \%$ VPO sample, $10 \mathrm{wt} \%$ conductive carbon, and $10 \mathrm{wt} \%$ polyvinylidene fluoride onto graphite substrate $\left(1 \mathrm{~cm}^{2}\right)$, and drying in a vacuum oven at $80^{\circ} \mathrm{C}$ overnight. The mass loading of active material is fixed at around $1 \mathrm{mg} .1 \mathrm{M} \mathrm{H}_{2} \mathrm{SO}_{4}$ solution was used as electrolyte.

The cyclic voltammetry (CV) curves were recorded in the potential window of $0.0-1.0 \mathrm{~V}$ at various scan rates ranging from 2 to $100 \mathrm{mV} \mathrm{s}^{-1}$. The galvanostatic charge-discharge profiles were collected in the same potential range at different constant current densities $(0.5-$ $20 \mathrm{~A} \mathrm{~g}^{-1}$ ). The specific capacitance of the electrode materials can be calculated from the charge-discharge data using the following equation:

$C=\frac{I \times \Delta t}{m \times \Delta V}$

where $C$ is the specific capacitance (F.g $\left.{ }^{-1}\right), m$ is the mass of the active material $(\mathrm{g}), \Delta t$ is the discharge time (s), $\Delta V$ is the potential window $(\mathrm{V})$, and $I$ is the current (A). The electrochemical impedance spectroscopy (EIS) measurement was carried out at open-circuit potential in the frequency range of $10^{5}-10^{-2} \mathrm{~Hz}$ with an applied perturbation signal of $5 \mathrm{mV}$.

\section{Results and discussion}

The low-angle X-ray diffraction (XRD) pattern of the gel-like precursor prepared in the presence of P123 is shown in Fig. 1, presenting three well-defined diffraction peaks. A fairly intense 100 peak is monitored at $2 \theta=0.68^{\circ}\left(d_{100}=12.97 \mathrm{~nm}\right)$, with additional higher order 110 and 200 peaks at $1.16^{\circ}$ and $1.36^{\circ}$, respectively. All these reflections can be assigned to the periodic $2 \mathrm{D}$ hexagonal mesostructure of the precursor, laying a solid foundation for the formation of mesoporous VPO material after post-synthesis heat treatment. Upon calcination, the precursor species reacted with each other to produce vanadium phosphate surrounding the ordered mesostructured framework assembled by the P123 micelles (Scheme 1). Eventually with the removal of the template by calcination, well-defined mesopores were introduced into the structure. The wide-angle XRD pattern of the obtained VPO sample is shown in Fig. 2a. The main distinct diffraction peaks can be well indexed to the known $\delta$-phase of $\mathrm{VOPO}_{4}$ (JCPDS No. 47-0951) [24,38]. $\delta$ - $\mathrm{VOPO}_{4}$ is generally reported to have a layered structure, built up from planes of $\mathrm{VO}_{6}$ octahedra linked by independent $\mathrm{PO}_{4}$ tetrahedra through sharing vertices $[39,40]$, as depicted in the inset of Fig. 2a. The average 

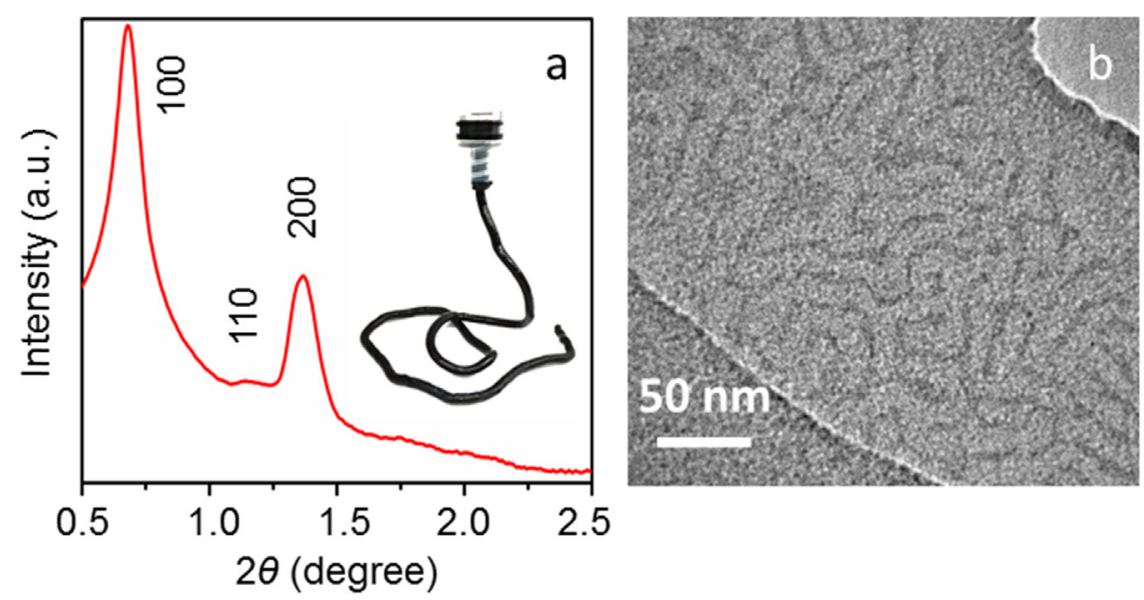

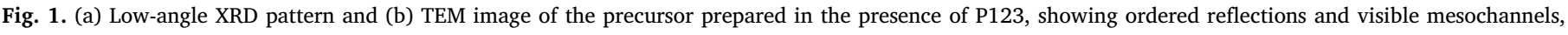
respectively. The photograph of the highly viscous LLC is shown in the inset of Fig. 1 (a).

crystallite size of the VPO sample is estimated to be around $8 \mathrm{~nm}$, by using the Scherrer equation $(D=0.9 \lambda /(\beta \cos \theta))$, where $D$ is the mean size of the crystalline domains ( $\AA$ ), $\lambda$ is the X-ray wavelength $\left(\mathrm{Cu} \mathrm{K} \mathrm{K}_{\alpha}=\right.$ $1.5406 \AA$ ),$\beta$ is the full width at half-maximum (FWHM) in radians, and $\theta$ is the Bragg diffraction angle. The visible peak broadening of the $\delta$ $\mathrm{VOPO}_{4}$ is indicative of the formation of nanoscale morphology, which has been verified in the latter part. The low-angle XRD pattern of the obtained VPO sample is presented in Fig. 2b. One single broad peak can be observed at around $1.71^{\circ}$ with no higher order peaks, revealing the existence of a less-ordered mesostructure [41]. In other words, the longrange ordering of the parent mesostructure has been disrupted to some extent after the calcination of the precursor. The single broad peak in the low-angle region is relevant to the characteristic distribution maximum of the nearest-neighbor pore-center-to-pore-center distance between the disordered pores [42]. The peak at about $2 \theta=1.71^{\circ}$ for the VPO sample corresponds to an interpore separation of $5.16 \mathrm{~nm}$. In comparison, the bulk VPO sample prepared in the absence of P123 does not exhibit any diffraction peaks in the low-angle XRD pattern (Fig. S1), which indicates the crucial role of the P123 template for inducing the formation of mesopores.

The morphology of the VPO sample was examined by scanning electron microscope (SEM). The representative SEM images of the VPO sample prepared in the presence of P123 are shown in Fig. 3a-b, showing uniform sheet-like morphology with an average thickness of $8-10 \mathrm{~nm}$ and a lateral size of several hundred nanometers. While for the bulk VPO material prepared in the absence of P123, the SEM images in Fig. S2 reveal its irregular morphology consisting of several aggregated particles. Transmission electron microscope (TEM) was utilized to provide direct and in-depth observations of the internal microstructure of the VPO nanosheets. A closer look at the mesostructure of the VPO nanosheets presents visible mesopores with diameters ranging between
4 and $5 \mathrm{~nm}$ (Fig. 3c-e). The HRTEM image in Fig. 3f exhibits well-resolved lattice fringes with an interplanar distance of $0.407 \mathrm{~nm}$, corresponding to the (111) crystal planes of $\delta$ - $\mathrm{VOPO}_{4}$. The selected-area electron diffraction (SAED) pattern in Fig. 3e represents a series of concentric rings made of small bright spots, revealing the polycrystalline nature of the VPO nanosheets [43]. The interplanar spacings calculated from the characteristic SAED patterns can be assigned to the (020), (031) and (040) lattice planes of $\delta$ - $\mathrm{VOPO}_{4}$, respectively. The elemental mapping (Fig. S3a) clearly displays the homogeneous distribution of vanadium, phosphorus and oxygen throughout the selected region of the VPO nanosheets, while the energy-dispersive X-ray (EDX) spectrum (Fig. S3b) gives a rough V/P atomic ratio of 1.1:1.0, which is in good agreement with the stoichiometric composition acquired from the crystallographic data. The bulk VPO was also checked by TEM for further comparison (Fig. S4), however no mesopores were detected throughout the specimen, which strongly suggests the pivotal templating function of P123 for constructing the mesoporous structure. The porosity of the VPO nanosheets was further characterized by $\mathrm{N}_{2}$ adsorption-desorption measurements. The obtained curve can be identified as a typical type IV isotherm with a hysteresis loop (Fig. S5a) [44]. The corresponding pore size distribution curve (Fig. S5b) obtained by the Barrett-Joyner-Halenda (BJH) method exhibits a sharp peak at $5 \mathrm{~nm}$ and a narrow peak at $\sim 2.5 \mathrm{~nm}$. The Brunauer-Emmett-Teller (BET) surface area is measured to be $\sim 18 \mathrm{~m}^{2} \mathrm{~g}^{-1}$. While, for the bulk VPO, no obvious hysteresis loop can be observed in the isotherm, showing a much lower BET surface area of $\sim 2 \mathrm{~m}^{2} \mathrm{~g}^{-1}$.

The skeletal bonding of the mesoporous VPO nanosheets was studied by Fourier transform infrared (FTIR) spectroscopy. The bands at around $3000-3600 \mathrm{~cm}^{-1}$ and the sharp band at $1618 \mathrm{~cm}^{-1}$ can be attributed to the surface-adsorbed water and hydroxyl groups,

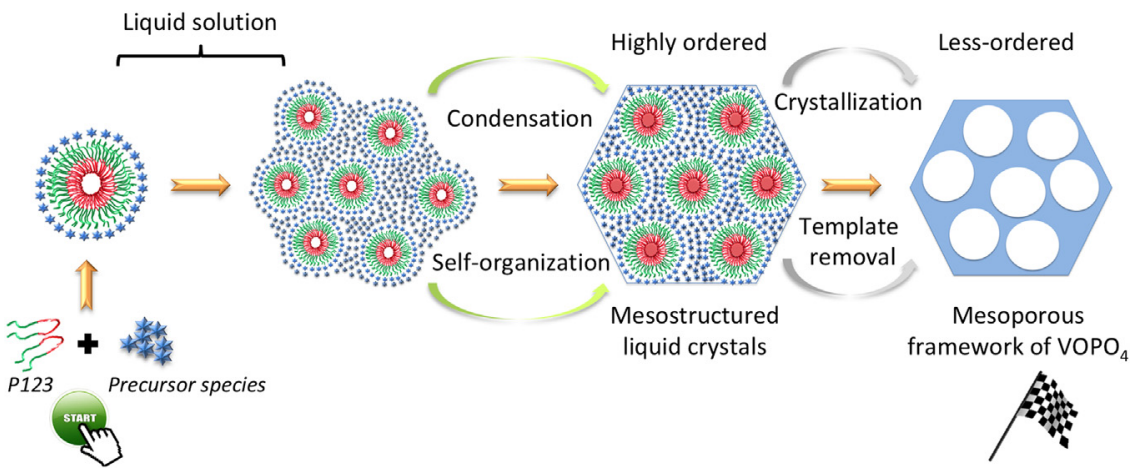

Scheme 1. Schematic illustration depicting the synthetic process of $\mathrm{VOPO}_{4}$ nanosheets with less-ordered mesoporous structure and crystallized frameworks. 

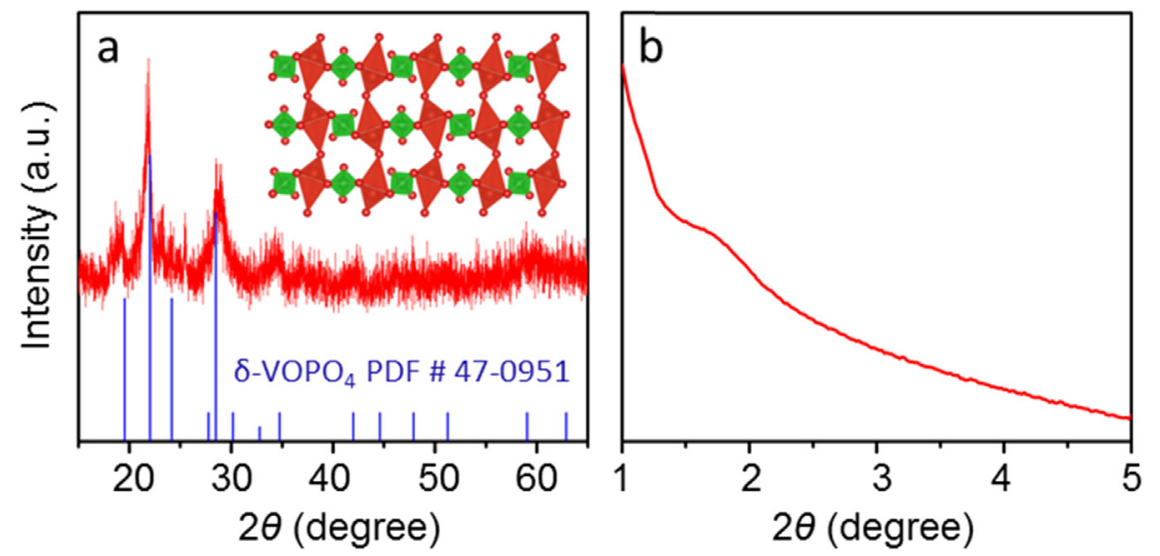

Fig. 2. (a) Wide-angle and (b) low-angle XRD patterns of the VPO sample prepared in the presence of P123.

respectively (Fig. 4a). The low energy region of the spectrum is enlarged in Fig. 4b. The pronounced band at $683 \mathrm{~cm}^{-1}$ can be credited to the characteristic V-O-P bending vibration [32,45-47]. A strong peak at $952 \mathrm{~cm}^{-1}$ and the adjacent shoulder at $994 \mathrm{~cm}^{-1}$ are attributed to the symmetric stretching mode of $\mathrm{PO}_{4}$ tetrahedral group, while the small peak at 1169 and intense peak at $1083 \mathrm{~cm}^{-1}$ originate from the antisymmetric stretching mode of $\mathrm{PO}_{4}$ group [48-51]. Additionally, one weak band is observed at $1037 \mathrm{~cm}^{-1}$, which may be assigned to the stretching vibration of $\mathrm{V}=\mathrm{O}$ group $[48,52,53]$.

Furthermore, the aging and calcination processes of the as-obtained precursor were monitored to shed some light on the possible formation steps of VPO via joint observation of FTIR and wide-angle XRD (Fig. S6 and S7). The FTIR spectra display diagnostic bands corresponding to phosphate species and surface species. The reaction between the
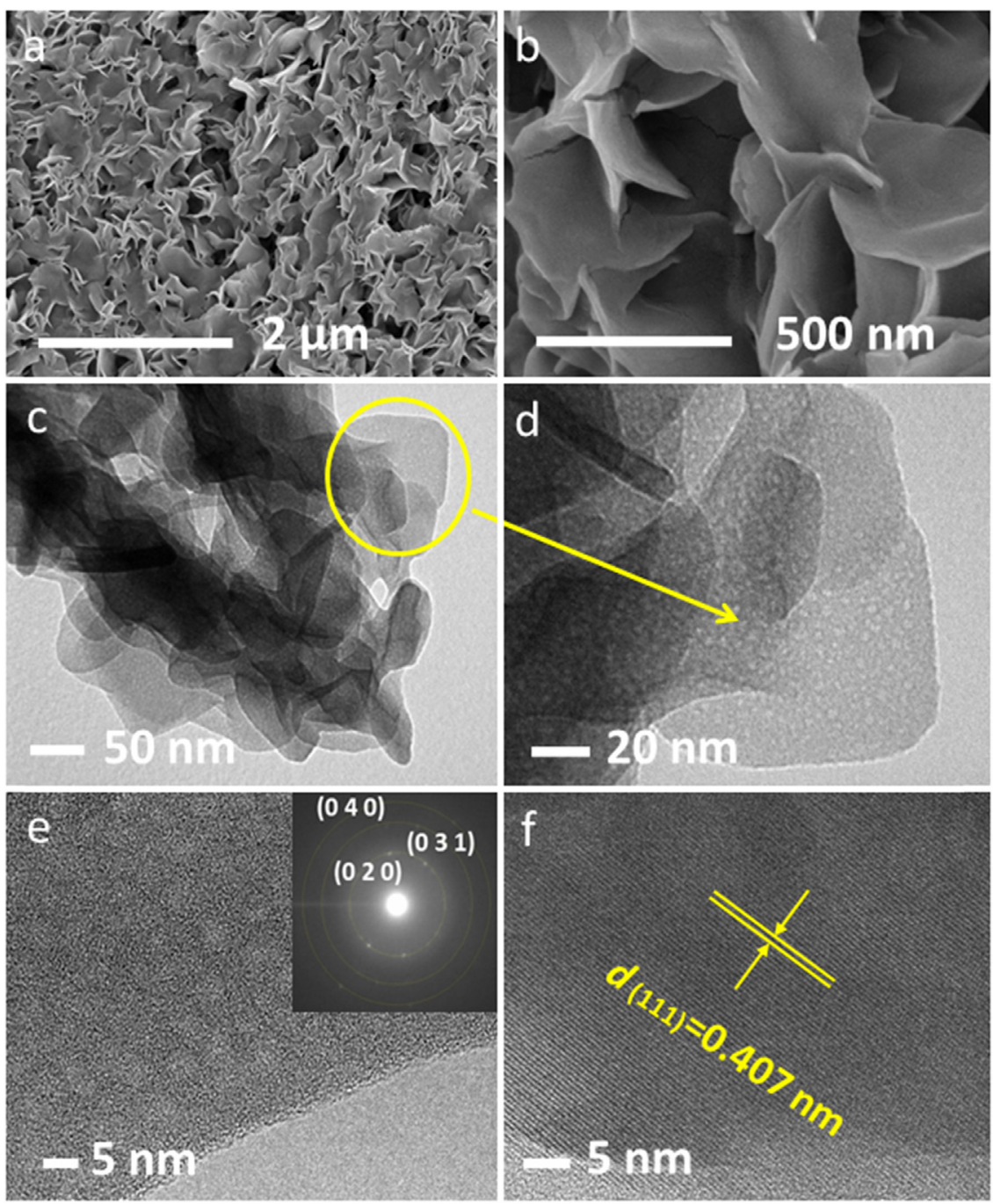

Fig. 3. (a, b) SEM images of the VPO sample prepared in the presence of P123 showing sheet-like morphology. (c, d) TEM images of the VPO nanosheets indicating the less-ordered mesoporous structure. (e, f) HRTEM image and SAED pattern of a single VPO nanosheet showing the polycrystalline nature. 

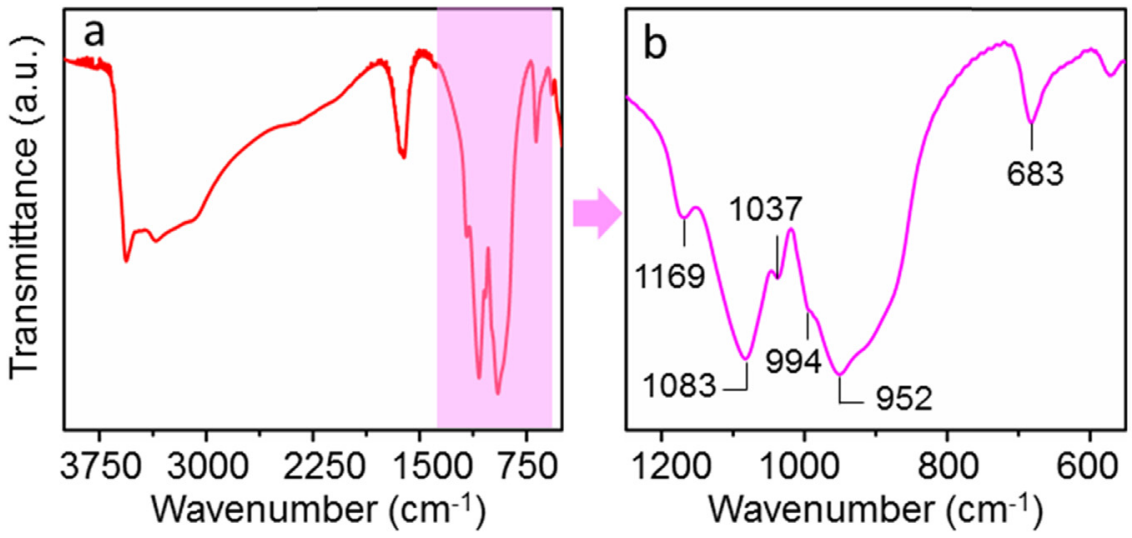

Fig. 4. FTIR spectra of the mesoporous VPO nanosheets.

vanadium precursor and phosphoric acid is an acid-base reaction that forms many intermediate species in the fresh gel-like precursor. The FTIR spectrum of the gel-like precursor displays a band at around $1720-1730 \mathrm{~cm}^{-1}$, which is due to the acetylacetonate group on the surface of the amorphous $\mathrm{VO}\left(\mathrm{H}_{x} \mathrm{PO}_{4}\right)_{n}$ species. This band becomes increasingly more intense at $150{ }^{\circ} \mathrm{C}$ and almost vanishes at around $250{ }^{\circ} \mathrm{C}$, indicating that acetylacetonate ions remain on the surface as a capping agent that have controlled the formation process. The surfactant signals (2500-2900 $\mathrm{cm}^{-1}$ and $1200-1400 \mathrm{~cm}^{-1}$ ) remain unchanged up to $150{ }^{\circ} \mathrm{C}$ but disappear at $250^{\circ} \mathrm{C}$. The phosphate region also does not show much changes up to $150^{\circ} \mathrm{C}$ and the intensity of the main broad band at $\sim 1000 \mathrm{~cm}^{-1}$ in the low energy region increases with further annealing. These observations reveal that the pre-formed acetylacetonate-capped amorphous intermediate species are stable up to $150{ }^{\circ} \mathrm{C}$. At $250^{\circ} \mathrm{C}$, the bands in the phosphate region become broadened and unresolved, indicating the presence of various phosphate species. The material remains amorphous up to $250{ }^{\circ} \mathrm{C}$ and forms a crystalline species at $350^{\circ} \mathrm{C}$, at which the phosphate region displays better resolved bands in the corresponding FTIR spectrum. While the FTIR spectra do not change significantly from $350^{\circ}$ to $450^{\circ} \mathrm{C}$, the XRD pattern of the sample calcined at $450^{\circ} \mathrm{C}$ corresponds well to nanocrystalline $\mathrm{VOPO}_{4}$. The sharp XRD line at $350^{\circ} \mathrm{C}$ may be due to $\mathrm{VOHPO}_{4} \cdot \mathrm{xH}_{2} \mathrm{O}$ species, which disappears with further heating to form $\mathrm{VOPO}_{4}$ (the weight loss of intercalated water molecules can be observed from the TG curve in Fig. S8). As seen from the spectral changes at various temperatures in the water region $\left(3000-3600 \mathrm{~cm}^{-1}\right)$, the water content of the samples increases up to $250^{\circ} \mathrm{C}$ and then, gradually decreases with further heating, thereby indicating the formation of many hydroxyl groups due to the presence of $\mathrm{HPO}_{4}{ }^{2-}$ and $\mathrm{H}_{2} \mathrm{PO}_{4}{ }^{-}$species in the pore walls. With further condensation of these species to form $\mathrm{VOPO}_{4}$, it is likely that the pore walls grow and crystallize into $\mathrm{VOHPO}_{4} \cdot \mathrm{xH}_{2} \mathrm{O}$ and transform into $\mathrm{VOPO}_{4}$ at higher temperatures. However, even at $450{ }^{\circ} \mathrm{C}$, many $\mathrm{V}-\mathrm{OH}$ or $\mathrm{P}-\mathrm{OH}$ species are still visible in the FTIR spectra. The sharp band at $\sim 3600 \mathrm{~cm}^{-1}$ is due to isolated O-H stretching vibration. With heating, they may not be accessible to water to form hydrogen bonding that broadens and shifts these signals to lower energy region.

The surface chemistry of the as-prepared mesoporous VPO nanosheets was investigated by X-ray photoelectron spectroscopy (XPS). The survey spectrum (Fig. 5a) ascertains the existence of vanadium, phosphorus, and oxygen in the sample. The calculated atomic ratio of $\mathrm{V} / \mathrm{P}$ is $1.0: 0.8$, which is very close to the stoichiometric ratio. The highresolution XPS spectrum of V 2p is shown Fig. 5b, representing a typical two-peak pattern with a spin-orbit splitting of $\sim 7.4 \mathrm{eV}$, as expected from $V 2 p_{3 / 2}$ and $V 2 p_{1 / 2}$ components of the doublet [54]. The binding energy (BE) associated with $\mathrm{V} 2 \mathrm{p}_{3 / 2}$ is commonly used to identify the valence states of vanadium species at the surface of the material [55]. As for the pristine mesoporous VPO, the intense $\mathrm{V} 2 \mathrm{p}_{3 / 2}$ peak is located at $518.0 \mathrm{eV}$, which is assignable to the $\mathrm{V}^{5+}$ species in vanadium phosphate compounds as reported [56-58]. The sharp peak centered at $133.9 \mathrm{eV}$ in Fig. 5 c originates from the P $2 p$ energy level and can be regarded as diagnostic of the pentavalent tetra-bonded phosphorus in phosphate materials $[56,59]$.

Herein, we have evaluated the electrochemical performance of the as-prepared mesoporous VPO nanosheets as electrode material for supercapacitors and compared their performance with bulk VPO. The capacitive behavior of the VPO sample was initially studied by cyclic voltammetry (CV) using a three-electrode configuration in $1 \mathrm{M} \mathrm{H}_{2} \mathrm{SO}_{4}$ electrolyte. Fig. 6a shows the first few cycles of CV curves of the mesoporous VPO electrode at a scan rate of $2 \mathrm{mV} \mathrm{s}^{-1}$ between 0.0 and $1.0 \mathrm{~V}$. Distinct from the quasi-rectangular $\mathrm{CV}$ response that is signature of double layer capacitance, the mesoporous VPO electrode exhibits distinguishable peak-shaped CV curves corresponding to typical pseudocapacitive behavior $[60,61]$. All the CV curves have completely coincided with each other throughout the initial cycling, with two pairs of pronounced redox peaks well preserved, suggesting the superb reversibility of the Faradaic redox reactions involved in the mesoporous VPO electrode.

As evidenced by XPS earlier, the only vanadium species in the pristine mesoporous VPO is $\mathrm{V}(\mathrm{V})$. Based on the E-pH diagram of the system V-O-H $[62,63]$, it is reasonable to infer that the two cathodic peaks here $(0.715 \mathrm{~V}, 0.372 \mathrm{~V})$ are presumably due to the reduction of $\mathrm{V}$ (V) to V(IV) and V(IV) to V(III), respectively, while the anodic peaks correspond to the reverse oxidation processes [64]. In support of our inference, we have conducted ex situ XPS analysis on the mesoporous VPO after being discharged to different cut-off voltages. Fig. S9a and b depict the high-resolution $\mathrm{V} 2 \mathrm{p}_{3 / 2}$ spectra of the VPO electrodes discharged to $0.6 \mathrm{~V}$ and $0.2 \mathrm{~V}$, respectively. The significant broadening of the $\mathrm{V} 2 \mathrm{p}_{3 / 2}$ peaks possibly indicates the presence of vanadium species with multiple valencies [65]. In order to separate the vanadium species into different chemical states, the $\mathrm{V} 2 \mathrm{p}_{3 / 2}$ peaks have been deconvoluted into several different contributions via Lorentzian-Gaussian fitting after Shirley background subtraction. In the case of the VPO electrode discharged to $0.6 \mathrm{~V}$ (Fig. S9a), it is noteworthy that the contribution with the lowest $\mathrm{BE}(516.4 \mathrm{eV})$ is assignable to the $\mathrm{V}^{4+} 2 \mathrm{p}_{3 / 2}$ component $[54,66]$, which provides positive evidence for the occurrence of reduction of $\mathrm{V}(\mathrm{V})$ to $\mathrm{V}(\mathrm{IV})$. Other two contributions with higher BEs $(518.0 \mathrm{eV}, 517.4 \mathrm{eV})$ can be attributed to the $\mathrm{V}^{5+} 2 \mathrm{p}_{3 / 2}$ components in different chemical environment: the former one is common in vanadium phosphate materials as described earlier; the latter one is more likely due to the vanadium oxide compounds from surface oxidation $[66,67]$. In the case of the VPO electrode discharged to $0.2 \mathrm{~V}$ (Fig. S9b), most notably, the contribution with the lowest $\mathrm{BE}(515.5 \mathrm{eV})$ is ascribable to the $\mathrm{V}^{3+} 2 \mathrm{p}_{3 / 2}$ component $[54,55,68]$, which offers strong support for our hypothesis about the further reduction of V(IV) to V(III). The rest of the contributions here have the same assignments with the former case. Thus, it can be reasonably concluded that the charge 

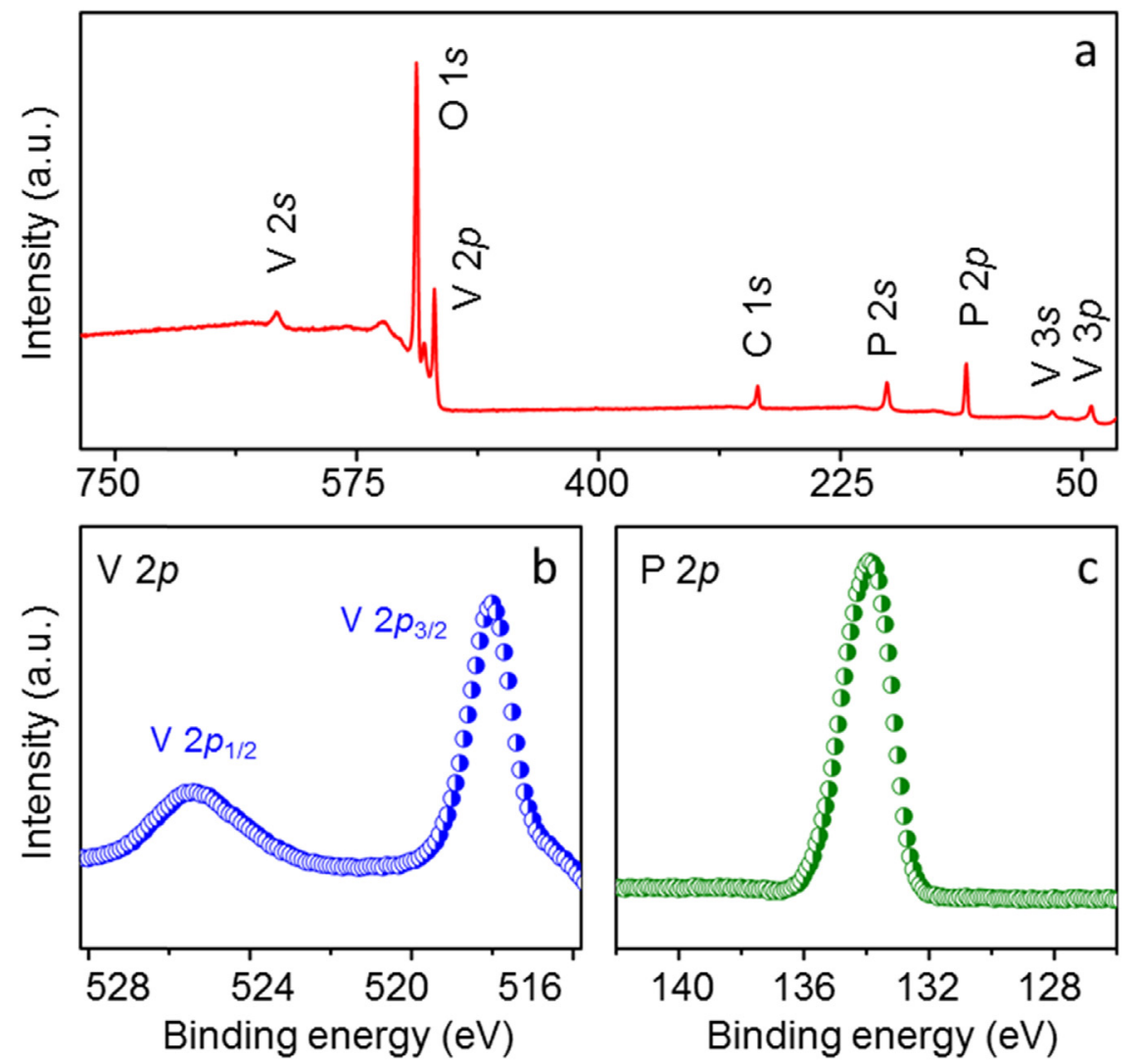

Fig. 5. XPS spectra of the as-prepared mesoporous VPO: (a) survey spectrum; (b) V 2p spectrum; (c) P 2p spectrum.

storage mechanism here should be based on the reversible two-step redox reactions between $\mathrm{V}(\mathrm{V})$ and $\mathrm{V}(\mathrm{III})$ in acidic medium, which is expected to provide excellent pseudocapacitance.

The cyclic voltammograms of the VPO electrodes were further recorded at various scan rates from 2 to $100 \mathrm{mV} \mathrm{s}^{-1}$ (Fig. S10). The mesoporous and bulk VPO electrodes exhibit nearly identical CV shape and peak positions, indicating the same possible redox reaction mechanism in both cases. It is noted that the peak current augments with
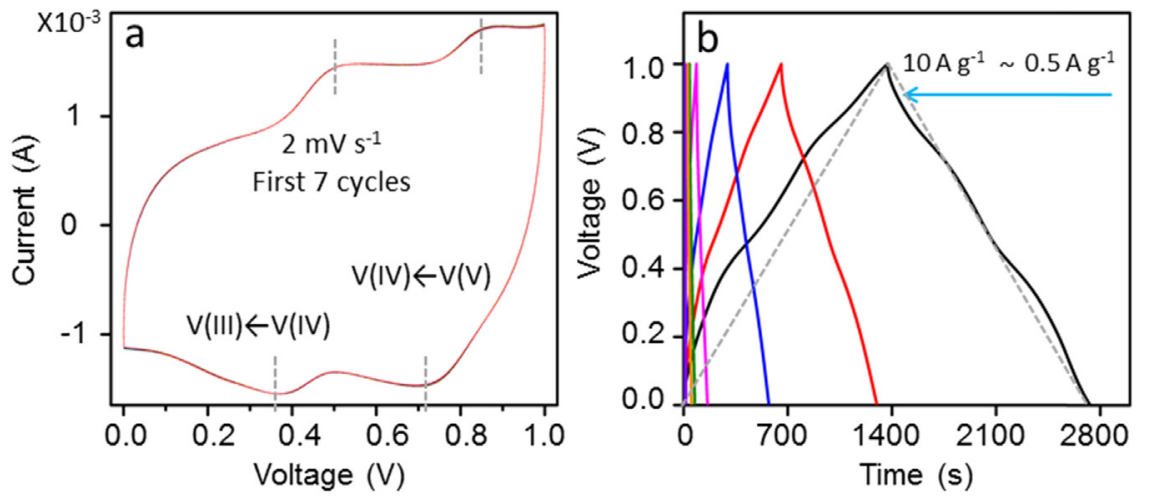

Fig. 6. (a) Cyclic voltammograms (first 7 cycles) of the mesoporous VPO at a scan rate of $2 \mathrm{mV} \mathrm{s}^{-1}$ in the voltage window of $0.0-1.0 \mathrm{~V}$; Galvanostatic charge-discharge curves of (b) mesoporous VPO and (c) bulk VPO at various current densities from 0.5 to $10 \mathrm{Ag}^{-1}$; (d) Comparison of the specific capacitances of mesoporous VPO (red bar) and bulk VPO (blue bar) at each rate.
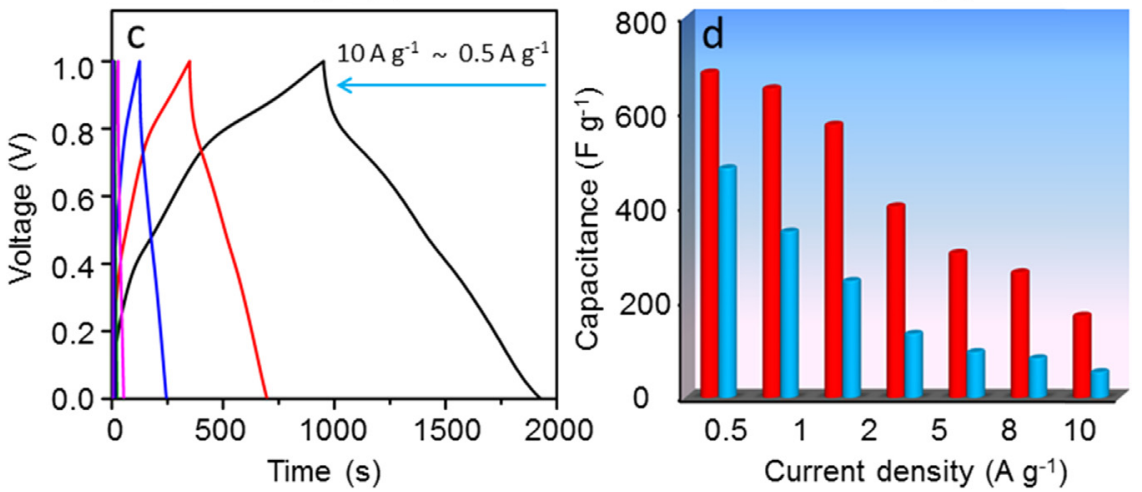
Table 1

Comparison of the supercapacitive performance of the as-prepared mesoporous VPO nanosheets with previously reported phosphate materials.

\begin{tabular}{|c|c|c|c|c|c|}
\hline Sample & Potential window (V) & Current density $\left(\mathrm{A} \mathrm{g}^{-1}\right)$ & Specific capacitance $\left(\mathrm{F}^{-1}\right)$ & Electrolyte & Ref. \\
\hline Vanadyl phosphate /reduced graphene oxide nanosheet & $-0.5-0.3$ & $\begin{array}{l}0.5 \\
10\end{array}$ & $\begin{array}{l}205 \\
139\end{array}$ & $0.5 \mathrm{M} \mathrm{K}_{2} \mathrm{SO}_{4}$ & [31] \\
\hline $\mathrm{VOPO}_{4}$-graphene Nanocomposites & $0.0-0.8$ & $\begin{array}{l}0.5 \\
10\end{array}$ & $\begin{array}{l}528 \\
<300\end{array}$ & $6.0 \mathrm{M} \mathrm{KOH}$ & [33] \\
\hline Vanadyl phosphate/graphene composites & $-0.1-0.9$ & $\begin{array}{l}0.5 \\
10\end{array}$ & $\begin{array}{l}508 \\
359\end{array}$ & $1.0 \mathrm{M} \mathrm{H}_{2} \mathrm{SO}_{4}$ & [47] \\
\hline Manganese phosphate/graphene foam composite & $0.0-0.4$ & $\begin{array}{l}0.5 \\
10\end{array}$ & $\begin{array}{l}270 \\
<50\end{array}$ & $6.0 \mathrm{M} \mathrm{KOH}$ & [71] \\
\hline Porous nano/microstructured cobalt pyrophosphate & $0.0-0.4$ & $\begin{array}{l}0.625 \\
6.25\end{array}$ & $\begin{array}{l}367 \\
237\end{array}$ & $3.0 \mathrm{M} \mathrm{KOH}$ & [72] \\
\hline Maricite-NaNiPO 4 nanoparticles & $0.0-0.55$ & $\begin{array}{l}1 \\
5\end{array}$ & $\begin{array}{l}368 \\
<250\end{array}$ & $1.0 \mathrm{M} \mathrm{NaOH}$ & [73] \\
\hline Hierarchical $\mathrm{NH}_{4} \mathrm{CoPO}_{4} \cdot \mathrm{H}_{2} \mathrm{O}$ microrods & $0.0-0.4$ & $\begin{array}{l}0.625 \\
6.25\end{array}$ & $\begin{array}{l}369 \\
306\end{array}$ & $3.0 \mathrm{M} \mathrm{KOH}$ & [74] \\
\hline Mesoporous VPO nanosheets & $0.0-1.0$ & $\begin{array}{l}0.5 \\
10\end{array}$ & $\begin{array}{l}767 \\
283\end{array}$ & $1.0 \mathrm{M} \mathrm{H}_{2} \mathrm{SO}_{4}$ & This work \\
\hline
\end{tabular}

the scan rate, implying that the charge storage process involves semiinfinite diffusion controlled redox reactions [69]. Furthermore, the integral area of the CV curves of the mesoporous VPO is always higher than that of the bulk VPO at each scan rate, suggesting the enhanced charge storage capability of the as-prepared mesoporous VPO.

The galvanostatic charge-discharge measurements were performed for both mesoporous VPO and bulk VPO at different current densities in the same electrode system and voltage window, as depicted in Fig. 6b-c. The non-linear charge-discharge curves could be a hint that the charge is stored in electrode based on Faradaic redox reactions, reconfirming the pseudocapacitive nature of the VPO materials in the present condition $[47,69,70]$. Additionally, the observed two pairs of charge and discharge plateaus remain consistent with the redox peaks in CV curves, further demonstrating the reversibility of the two-step redox reactions involved in the charge-discharge process. Fig. $6 \mathrm{~d}$ compares the calculated specific capacitances of the two VPO electrodes. In the case of the mesoporous VPO, the specific capacitance can reach up to $767 \mathrm{~F} \mathrm{~g}^{-1}$ at $0.5 \mathrm{~A} \mathrm{~g}^{-1}$. With the increase in current density, the mesoporous VPO is still capable of delivering appreciable capacitances of 718, 624, 434, 328 , and $283 \mathrm{~F} \mathrm{~g}^{-1}$ at $1,2,5,8,10 \mathrm{Ag}^{-1}$, respectively. While for the bulk VPO, the corresponding capacitance values are merely 481, 348, $245,134,96$, and $83 \mathrm{Fg}^{-1}$ at the same rates. The above results show that our mesoporous VPO can easily outperform its bulk counterpart in both capacitance and rate capability. Furthermore, the superior pseudocapacitive performance of our mesoporous VPO also makes it stand out from many reported phosphate materials, even comparable to some VPO/graphene composites (Table 1) [31,33,47,71-74]. Durability is another important criterion for evaluating the suitability of electrode candidates for supercapacitors. Fig. S11 presents the long-term cycling stability of the mesoporous VPO at $100 \mathrm{mV} \mathrm{s}^{-1}$. Satisfactorily, the capacitance retention of the mesopores VPO has almost remained at around $100 \%$ and the original CV shape is well preserved throughout the 4000 repeated cycles, indicating the good cycling performance and remarkable structural stability of the material.

The electrochemical impedance spectroscopy (EIS) study was conducted to gain deeper insights into the electrochemical behavior of the VPO samples. Similar Nyquist plots were acquired for our mesoporous VPO and the bulk VPO, as illustrated in Fig. S12. In the high-to-medium frequency region, the depressed semicircle is correlated with the charge-transfer resistance $\left(R_{\mathrm{ct}}\right)$ [75]. The smaller semicircle diameter of the mesoporous VPO indicates that its $R_{\mathrm{ct}}$ is definitely lower than its bulk counterpart, which is favorable for fast Faradaic redox reactions. While in the low frequency area, the inclined straight line is correlated to the ion diffusion process $[76,77]$. Generally, the deviation from an ideal vertical line to phase angles below $90^{\circ}$ is regarded as an indication of typical pseudocapacitive behavior [78]. In addition, the greater slope of the mesoporous VPO indicates its much lower diffusion resistance, which facilitates the rapid diffusion of electrolyte ions to the active sites in the electrode [69].

The excellent pseudocapacitive performance of our mesoporous VPO may be attributed to the favorable mesoporous architecture with nanosheet morphology, as well as its rich redox behavior. The reversible electrochemical transfer reactions between $\mathrm{V}(\mathrm{V})$ and $\mathrm{V}(\mathrm{III})$ fundamentally determine the increased charge storage capability. The incorporation of mesopores can give rise to abundant accessible active sites and afford a high infiltration capacity for electrolyte, and hence, ensure the sufficient transport of ions/solvent to the active sites, thus significantly improving its pseudocapacitance. Furthermore, the uniform nanosheet morphology together with interconnected mesopores can cooperatively reinforce the electron transfer and ion diffusion (as evidenced by EIS analysis) during the charge-discharge process, and thereby, greatly improve the rate performance.

\section{Conclusion}

We have demonstrated the feasibility of LLC directed-synthesis of mesoporous vanadium phosphate for the first time. The as-prepared $\mathrm{VOPO}_{4}$ nanosheets exhibit less-ordered mesoporous architectures with crystallized frameworks and are free of surfactant impurities in their composition. As a prospective electrode material for supercapacitors, the as-prepared mesoporous $\mathrm{VOPO}_{4}$ nanosheets exhibit excellent pseudocapacitive performance $\left(767 \mathrm{Fg}^{-1}\right.$ at $\left.0.5 \mathrm{Ag}^{-1}\right)$ by virtue of their mesoporous architectures and reversible two-step redox reaction mechanism. It is expected that the proposed LLC-templating strategy will open up a new path for the easy and reproducible synthesis of various mesoporous metal phosphates with superior electrochemical performance for energy storage devices.

\section{Acknowledgement}

This work was supported by the Australian Research Council (ARC) Future Fellow (FT150100479), JSPS KAKENHI (17H05393 and 17K19044), and the research fund by the Suzuken Memorial Foundation.

\section{Appendix A. Supplementary material}

Supplementary data associated with this article can be found in the online version at doi:10.1016/j.nanoen.2018.07.052.

\section{References}

[1] M.E. Davis, Nature 417 (2002) 813-821.

[2] N. Linares, A.M. Silvestre-Albero, E. Serrano, J. Silvestre-Albero, J. Garcia- 
Martinez, Chem. Soc. Rev. 43 (2014) 7681-7717.

[3] W. Chaikittisilp, K. Muraoka, Q.M. Ji, K. Ariga, Y. Yamauchi, J. Mater. Chem. A 2 (2014) 12096-12103.

[4] Y. Liu, R. Che, G. Chen, J. Fan, Z. Sun, Z. Wu, M. Wang, B. Li, J. Wei, Y. Wei, G. Wang, G. Guan, A.A. Elzatahry, A.A. Bagabas, A.M. Al-Enizi, Y. Deng, H. Peng, D. Zhao, Sci. Adv. 1 (2015) 1500166.

[5] B.Y. Guan, L. Yu, X.W. Lou, J. Am. Chem. Soc. 138 (2016) 11306-11311.

[6] H. Hu, Bu.Y. Guan, Xiong W. Lou, Chem 1 (2016) 102-113.

[7] B. Liu, H.Q. Peng, C.N. Ho, H. Xue, S. Wu, T.W. Ng, C.S. Lee, W. Zhang, Small 13 (2017) 1701875.

[8] M. Zhang, L. Zhang, Y. Chen, L. Li, Z. Su, C. Wang, Chem. Sci. 8 (2017) 8067-8077.

[9] B.Y. Guan, S.L. Zhang, X.W. Lou, Angew. Chem. Int. Ed. 57 (2018) 6176-6180.

[10] J.S. Beck, J.C. Vartuli, W.J. Roth, M.E. Leonowicz, C.T. Kresge, K.D. Schmitt, C.T.W. Chu, D.H. Olson, E.W. Sheppard, S.B. McCullen, J.B. Higgins, J.L. Schlenker, J. Am. Chem. Soc. 114 (1992) 10834-10843.

[11] C.T. Kresge, M.E. Leonowicz, W.J. Roth, J.C. Vartuli, J.S. Beck, Nature 359 (1992) $710-712$.

[12] G.S. Attard, J.C. Glyde, C.G. Göltner, Nature 378 (1995) 366-368.

[13] G.S. Attard, P.N. Bartlett, N.R.B. Coleman, J.M. Elliott, J.R. Owen, J.H. Wang, Science 278 (1997) 838-840.

[14] Ö. Çelik, Ö. Dag, Angew. Chem. Int. Ed. 40 (2001) 3800-3803.

[15] Y. Yamauchi, A. Sugiyama, R. Morimoto, A. Takai, K. Kuroda, Angew. Chem. Int. Ed. 47 (2008) 5371-5373.

[16] Y. Türker, Ö. Dag, J. Mater. Chem. 18 (2008) 3467-3473.

[17] Y. Yamauchi, A. Tonegawa, M. Komatsu, H. Wang, L. Wang, Y. Nemoto, N. Suzuki, K. Kuroda, J. Am. Chem. Soc. 134 (2012) 5100-5109.

[18] S. Makino, Y. Yamauchi, W. Sugimoto, J. Power Sources 227 (2013) 153-160.

[19] M. Conte, G. Budroni, J.K. Bartley, S.H. Taylor, A.F. Carley, A. Schmidt, D.M. Murphy, F. Girgsdies, T. Ressler, R. Schlögl, G.J. Hutchings, Science 313 (2006) 1270-1273.

[20] G.J. Hutchings, J. Mater. Chem. 14 (2004) 3385-3395.

[21] L. Beneš, K. Melánová, J. Svoboda, V. Zima, J. Incl. Phenom. Macrocycl. Chem. 73 (2012) 33-53.

[22] N.G. Park, K.M. Kim, S.H. Chang, Electrochem. Commun. 3 (2001) 553-556.

[23] N. Dupré, J. Gaubicher, T. Le Mercier, G. Wallez, J. Angenault, M. Quarton, Solid State Ion. 140 (2001) 209-221.

[24] B.M. Azmi, T. Ishihara, H. Nishiguchi, Y. Takita, J. Power Sources 119-121 (2003) 273-277.

[25] B. Zhang, Y.D. Han, J.C. Zheng, J.F. Zhang, C. Shen, L. Ming, X.B. Yuan, H. Li, Chem. Commun. 50 (2014) 11132-11134.

[26] M. Zhang, S. Zhang, H. Gao, F.L. Meng, C. Deng, J. Electroanal. Chem. 713 (2014) 119-124.

[27] G. He, W.H. Kan, A. Manthiram, Chem. Mater. 28 (2016) 682-688.

[28] Y. Zhu, L. Peng, D. Chen, G. Yu, Nano Lett. 16 (2016) 742-747.

[29] L. Peng, Y. Zhu, X. Peng, Z. Fang, W. Chu, Y. Wang, Y. Xie, Y. Li, J.J. Cha, G. Yu, Nano Lett. 17 (2017) 6273-6279.

[30] C. Wu, X. Lu, L. Peng, K. Xu, X. Peng, J. Huang, G. Yu, Y. Xie, Nat. Commun. 4 (2013) 1-7.

[31] Y. He, X. Yang, Y. Bai, J. Zhang, L. Kang, Z. Lei, Z.H. Liu, Electrochim. Acta 178 (2015) 312-320.

[32] Z. Luo, E. Liu, T. Hu, Z. Li, T. Liu, Ionics 21 (2015) 289-294.

[33] K.H. Lee, Y.W. Lee, S.W. Lee, J.S. Ha, S.S. Lee, J.G. Son, Sci. Rep. 5 (2015) 13696.

[34] I.E. Rauda, V. Augustyn, B. Dunn, S.H. Tolbert, Acc. Chem. Res. 46 (2013) 1113-1124.

[35] T. Doi, T. Miyake, Chem. Commun. 14 (1996) 1635-1636.

[36] J. El Haskouri, M. Roca, S. Cabrera, J. Alamo, A. Beltrán-Porter, D. Beltrán-Porter, M.D. Marcos, P. Amorós, Chem. Mater. 11 (1999) 1446-1454.

[37] M.A. Carreon, V.V. Guliants, Microporous Mesoporous Mater. 55 (2002) 297-304.

[38] B.M. Azmi, T. Ishihara, H. Nishiguchi, Y. Takita, Electrochim. Acta 48 (2002) $165-170$.

[39] N. Dupré, J. Gaubicher, J. Angenault, G. Wallez, M. Quarton, J. Power Sources 97-98 (2001) 532-534.

[40] F. Girgsdies, M. Schneider, A. Brückner, T. Ressler, R. Schlögl, Solid State Sci. 11 (2009) 1258-1264.

[41] G.S. Attard, J.M. Corker, C.G. Göltner, S. Henke, R.H. Templer, Angew. Chem. Int. Ed. 36 (1997) 1315-1317.

[42] S.K. Das, M.K. Bhunia, A.K. Sinha, A. Bhaumik, ACS Catal. 1 (2011) 493-501.

[43] R.F. Egerton, Physical Principles of Electron Microscopy: an Introduction to TEM, SEM, and AEM, Springer US, 2006.

[44] M. Kruk, M. Jaroniec, Chem. Mater. 13 (2001) 3169-3183.

[45] R.N. Bhargava, S.R.A. Condrate, Appl. Spectrosc. 31 (1977) 230-236.

[46] M.R. Antonio, R.L. Barbour, P.R. Blum, Inorg. Chem. 26 (1987) 1235-1243.

[47] N. Chen, J. Zhou, Q. Kang, H. Ji, G. Zhu, Y. Zhang, S. Chen, J. Chen, X. Feng, W. Hou, J. Power Sources 344 (2017) 185-194.

[48] P. Borah, A. Datta, Appl. Catal. A 376 (2010) 19-24.

[49] K. Zhou, Z. Gui, Y. Hu, RSC Adv. 6 (2016) 100344-100351.

[50] A. Datta, S. Sakthivel, M. Kaur, A.M. Venezia, G. Pantaleo, A. Longo, Microporous Mesoporous Mater. 128 (2010) 213-222.

[51] K. Melánová, L. Beneš, V. Zima, E. Černošková, J. Brus, M. Urbanová, M. Trchová, J. Dybal, Eur. J. Inorg. Chem. 2007 (2007) 444-451.

[52] L. Beneš, V. Zima, K. Melánová, M. Trchová, P. Čapková, B. Koudelka, P. Matějka, Chem. Mater. 14 (2002) 2788-2795.

[53] M. Trchová, P. Čapková, P. Matějka, K. Melánová, L. Beneš, E. Uhlířová, J. Solid State Chem. 148 (1999) 197-204.

[54] J.F. Moulder, J. Chastain, Handbook of X-ray Photoelectron Spectroscopy: A Reference Book of Standard Spectra for Identification and Interpretation of XPS
Data, Physical Electronics Division, Perkin-Elmer Corporation, 1992.

[55] G. Silversmit, D. Depla, H. Poelman, G.B. Marin, R. De Gryse, J. Electron. Spectrosc Relat. Phenom. 135 (2004) 167-175.

[56] L.M. Cornaglia, E.A. Lombardo, Appl. Catal. A 127 (1995) 125-138.

[57] P. Delichère, K.E. Béré, M. Abon, Appl. Catal. A 172 (1998) 295-309.

[58] M. Abon, K.E. Bere, A. Tuel, P. Delichere, J. Catal. 156 (1995) 28-36.

[59] M.P. Casaletto, L. Lisi, G. Mattogno, P. Patrono, G. Ruoppolo, G. Russo, Appl. Catal. A 226 (2002) 41-48.

[60] C. Costentin, T.R. Porter, J.M. Saveant, ACS Appl. Mater. Interfaces 9 (2017) 8649-8658.

[61] P. Simon, Y. Gogotsi, B. Dunn, Science 343 (2014) 1210-1211.

[62] M. Pourbaix, Atlas of Electrochemical Equilibria in Aqueous Solutions, National Association of Corrosion Engineers, 1974.

[63] N. Takeno, Atlas of Eh-pH Diagrams: Intercomparison of Thermodynamics Databases, National Institute of Advanced Industrial Science and Technology, 2005.

[64] A. Liu, M. Ichihara, I. Honma, H. Zhou, Electrochem. Commun. 9 (2007) 1766-1771.

[65] B.M. Reddy, P.M. Sreekanth, E.P. Reddy, Y. Yamada, Q. Xu, H. Sakurai, T. Kobayashi, J. Phys. Chem. B 106 (2002) 5695-5700.

[66] N. Ibris, A.M. Salvi, M. Liberatore, F. Decker, A. Surca, Surf. Interface Anal. 37 (2005) 1092-1104.

[67] V.I. Nefedov, M.N. Firsov, I.S. Shaplygin, J. Electron. Spectrosc. Relat. Phenom. 26 (1982) 65-78.

[68] P. Mei, M. Pramanik, J. Lee, T. Takei, Y. Ide, M.S. Hossain, J.H. Kim, Y. Yamauchi, Phys. Chem. Chem. Phys. 19 (2017) 9156-9163.

[69] R. Bendi, V. Kumar, V. Bhavanasi, K. Parida, P.S. Lee, Adv. Energy Mater. 6 (2016) 1501833.

[70] P. Mei, M. Pramanik, C. Young, Z. Huang, M.S.A. Hossain, Y. Sugahara, Y. Yamauchi, J. Mater. Chem. A 5 (2017) 23259-23266.

[71] A.M. Abdulmajid, J.M. Moshawe, M.M. Tshifhiwa, O.O. Kabir, B. Abdulhakeem, M. Ncholu, J. Colloid Interface Sci. 494 (2017) 325-337.

[72] H. Pang, Z. Yan, Y. Ma, G. Li, J. Chen, J. Zhang, W. Du, S. Li, J. Solid State Electrochem. 17 (2013) 1383-1391.

[73] B. Senthilkumar, K.V. Sankar, L. Vasylechko, Y.S. Lee, R.K. Selvan, RSC Adv. 4 (2014) 53192-53200.

[74] H. Pang, Z. Yan, W. Wang, J. Chen, J. Zhang, H. Zheng, Nanoscale 4 (2012) 5946-5953.

[75] G. Wang, L. Zhang, J. Zhang, Chem. Soc. Rev. 41 (2012) 797-828.

[76] L. Cui, J. Li, X.G. Zhang, J. Appl. Electrochem. 39 (2009) 1871-1876.

[77] W. Sun, R. Zheng, X. Chen, J. Power Sources 195 (2010) 7120-7125.

[78] V. Augustyn, P. Simon, B. Dunn, Energy Environ. Sci. 7 (2014) 1597-1614.

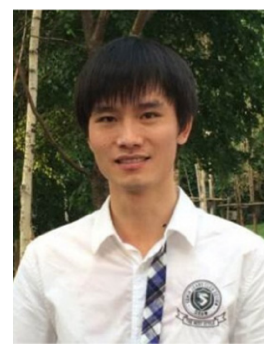

Peng Mei received his B.Eng. degree in Materials Chemistry from South-central University for Nationalities, China in 2012, and his M.Sc. degree in Physical Chemistry from Northeast Normal University, China in 2015. He is now a Ph.D. candidate under Professors Yamauchi's and Sugahara's supervision at Waseda University, and a junior researcher in National Institute for Materials Science (NIMS), Japan. His research focuses on templated-synthesis of mesoporous materials (e.g. phosphorus-based) and their application for energy storage and conversion.

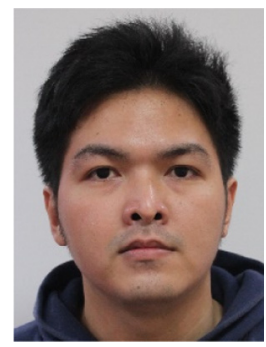

Yusuf Valentino Kaneti obtained his Ph.D. degree in Materials Science and Engineering from the University of New South Wales in 2014. He is currently working as a JSPS postdoctoral fellow in Professor Yamauchi's group at the International Center for Materials Nanoarchitectonics (WPI-MANA), National Institute for Materials Science (NIMS), Japan. His research focuses on the synthesis, characterization and application of porous nanomaterials for energy storage and gas sensors. He has published 50 papers in internationally-refereed journals with 1225 citations ( $h$-index of 18). 


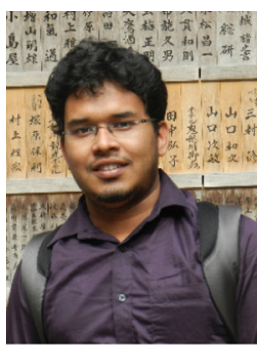

Malay Pramanik obtained his Ph.D. degree in 2014 from Indian Association for the Cultivation of Science (IACS), Kolkata, India. He joined Prof. Yamauchi Group in National Institute for Materials Science (NIMS), Japan in 2014 as a postdoctoral researcher. After working as a JSPS postdoctoral fellow in Prof. Yamauchi Group in 2017, Pramanik joined as senior research officer in HP Green R\&D center, Bangalore, India. His current research focuses on nanomaterials for lithium ion batteries and sodium ion batteries.

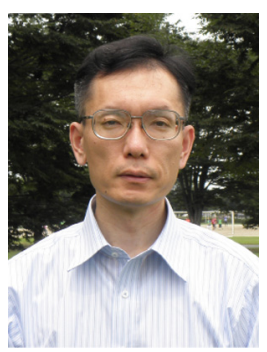

Toshiaki Takei received his Ph.D. degree in Chemistry from Gakushuin University, Japan in 2006. After graduation he researched biomaterials (e.g., designed polypeptide, hydrogel and protein) at Kobe University and the University of Tokyo, Japan. Now he is working in National Institute for Materials Science (NIMS, Japan) as transmission electron microscope (TEM) operator and his work contributes to characterization of nanomaterials with various compositions.

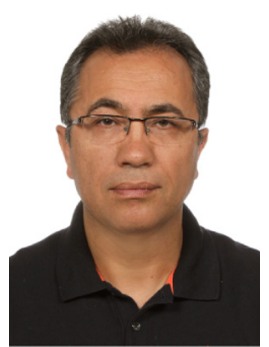

Ömer Dag received his B.Sc., M.Sc., and Ph.D. degrees in Chemistry from Middle East Technical University, Turkey in 1986, 1989, and 1994, respectively, and postdoctoral training from University of Toronto, Canada. He is a member of Science Academy, Istanbul, Turkey. His research focuses on the lyotropic liquid crystalline mesophases of salts-surfactants, acid-surfactants and their use in the synthesis of mesoporous materials towards various applications, such as gel electrolytes and mesoporous electrodes.

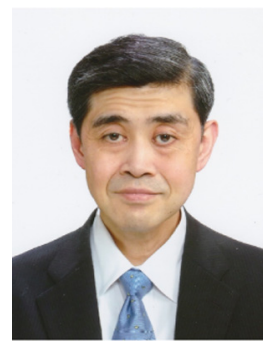

Yoshiyuki Sugahara is professor in Faculty of Science and Engineering at Waseda University. He received his Ph.D. degree in Applied Chemistry from Waseda University (1988). He joined Waseda University as a faculty member in 1990 after his postdoctoral study at Massachusetts Institute of Technology (1989-90). His research interests include preparation of metal oxide nanostructures (nanoparticles and nanosheets) and their surface modification with organic compounds, application of surface-modified nanostructures to organic-inorganic hybrid materials, preparation of organic-inorganic hybrid materials via the solgel process and polymer-derived non-oxide ceramics.

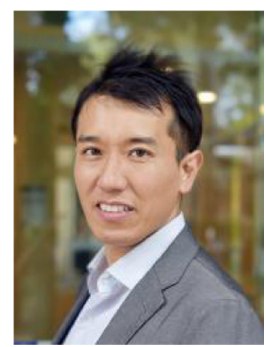

Yusuke Yamauchi received his Ph.D. degree from Waseda University (2007), Japan. After that, he joined NIMS to start his own research group. From 2018, he joined the University of Queensland (Australia) as a full-time professor. He concurrently serves as an honorary group leader of NIMS, a visiting professor at several universities (Waseda Univ, Qingdao Univ. of Sci. and Technol., Kyung Hee Univ, Tianjin Univ., King Saud Univ., etc), and an associate editor of Journal of Materials Chemistry A. He has published over 550 papers in international refereed journals with $\sim 30,000$ citations (h-index $\sim 80$ ). He was selected as one of the Highly-Cited Researchers in Chemistry in 2016 and 2017. 